\title{
1 Salmonella Typhimurium infection drives NK cell loss and conversion to 2 ILC1-like cells, and CIS inhibition enhances antibacterial immunity
}

4 Timothy R. McCulloch ${ }^{1}$, Gustavo R. Rossi ${ }^{1}$, Timothy J. Wells ${ }^{1,2}$, Fernando Souza5 Fonseca-Guimaraes ${ }^{1}$

61 University of Queensland Diamantina Institute, The University of Queensland, 7 Woolloongabba, QLD 4102, Australia.

$8{ }^{2}$ Australian Infectious Diseases Research Centre, University of Queensland, Brisbane 4072, 9 QLD, Australia

10 Correspondence:

11 Fernando S. F. Guimaraes

12 f.guimaraes@uq.edu.au

13 Abstract

Keywords: CIS, TGF-bR signaling, anti-bacterial responses, natural killer cells, innate

Immunotherapy has revolutionized cancer therapy by reactivating tumor-resident cytotoxic lymphocytes. More recently, immunotherapy has emerged to restore immunity against infectious agents, including bacterial infections. Immunotherapy primarily targets inhibitory pathways in tumor-resident $\mathrm{T}$ cells, however interest in other effector populations, such as natural killer (NK) cells, is growing. We have previously discovered that NK cell metabolism, proliferation, and activation can be neutralized through the TGF- $\beta$ immunosuppressive pathway by inducing plasticity of NK cells and differentiation into ILC1like subsets. NK cells are also regulated through cytokine-inducible SH2-containing protein (CIS), which is induced by IL-15 and is a potent intracellular checkpoint suppressing NK cell survival and function. Targeting these two distinct pathways to restore NK cell function has shown promise is cancer models, but their application in bacterial infection remains unknown. Here, we investigate whether enhancement of NK cell function can improve anti-bacterial immunity, using Salmonella Typhimurium as a model. We identified conversion of NK cells to ILC1-like for the first time in the context of bacterial infection, however TGF- $\beta$ signaling was curiously redundant in this plasticity. Future work should focus on identifying drivers of ILC1 plasticity and its functional implication in bacterial infection models. We further describe that CIS-deficient mice displayed enhanced pro-inflammatory function and dramatically enhanced anti-infection immunity. Inhibition of CIS may present as a viable therapeutic option to enhance immunity towards bacterial infection. 


\section{Introduction}

Natural killer (NK) cells are cytotoxic innate lymphocytes which have well described roles in the host defense against viral pathogens and cancer. Yet their ability to contribute to immunity in many bacterial infections, including Salmonella enterica serovar Typhimurium, remains unclear. NK cells have the potential to promote anti-Salmonella immunity through direct killing of infected cells and activation of infected cells through proinflammatory cytokine production, namely interferon (IFN)- $\gamma$. Previous studies in a murine model of $S$. Typhimurium infection have indicated that NK cells can contribute to protective IFN- $\gamma$ and disease clearance in immunocompromised mice, but are otherwise dispensable when $\mathrm{CD}^{+} \mathrm{T}$ cells are present (Kupz et al., 2013). The idea of NK cell redundancy when adaptive lymphocytes are present has also been suggested in human disease (Vély et al., 2016). This suggests that the standard depletion model using anti-NK1.1 or anti-asialo-GM1 antibodies is insufficient to gauge the full potential of NK cells to promote antibacterial immunity in otherwise immunocompetent hosts. Depletion studies do not account for the impact of NK cell regulation during infection through the action of immunoregulatory cytokines such as interleukin (IL)-10 and transforming growth factor (TGF)- $\beta$, NK cell lymphopenia, and inhibitory immune checkpoints. Targeting these mechanisms of regulation may allow NK cells to participate in immunity where they may otherwise have been incapable. cancer therapy. The classical targets are surface immune checkpoint molecules, such as programmed cell death protein-1 (PD-1), and cytotoxic T-lymphocyte-associated protein-4 (CTLA-4), which bind to their ligand on infected or tumor cells to ablate lymphocyte function. Blockade of these receptors can reverse this immune suppression and restore lymphocyte function (Robert, 2020). However, interest is rising in targeting other types of molecules, including regulatory cytokines and intracellular immune checkpoints. TGF- $\beta$ is a pleiotropic 
62 cytokine of the TGF superfamily which has potent regulatory effects on NK cells by repressing mammalian target of rapamycin (mTOR) (Viel et al., 2016) and converting them to an innate

64 lymphoid cell (ILC)1-like phenotype (Gao et al., 2017). Also of particular importance in NK cells is cytokine-inducible SH2-containing protein (CIS), which acts as a negative regulator of IL-15 signaling to limit NK cell proliferation and pro-inflammatory function (Delconte et al.,

67 2016). Inhibition of these two pathways in bacterial infection may restore the function of NK cells, allowing them to contribute towards anti-bacterial immunity. difficult to treat, immunotherapy is arising as a potential alternative or conjunction to traditional antimicrobial therapy (McCulloch et al., 2021). In this study, we use a 'gain of function' model to investigate whether NK cell function could be enhanced or restored during infection to boost anti-bacterial immunity. Our group and others have focused on the improvement of NK cell function through the deletion of receptors for immunoregulatory molecules TGF- $\beta$ (Gao et al., 2017; Rautela et al., 2019; Viel et al., 2016) or CIS (Delconte et al., 2016). Here, we investigate whether simultaneous immune checkpoint suppression of TGF-

$77 \quad \beta$ and CIS signaling in a new transgenic mouse model could act synergistically to increase the magnitude of NK cell effector function against Salmonella infection. 


\section{$80 \quad$ Results}

\section{NK cells are depleted and converted to ILC1-like cells during $S$. Typhimurium infection}

84 human disease. At day 10 post infection, spleens and livers were removed to observe

85 phenotypic changes in immune cells. Of note, we observed a phenotypic switch from

86 conventional NK (cNK) cells to ILC1-like cells, as determined by upregulation of tissue

87 residence marker CD49a. This occurred in both the spleen (Fig. 1a,b) and liver (Fig. 1c,d) of

88 infected mice. Furthermore, we also observed significant NK cell lymphopenia in the blood

89 (Fig. 1e) and spleen (Fig. 1f) of infected mice compared to uninfected. NK cell lymphopenia

90 was not seen in the liver of infected mice, where normal NK numbers were persevered (Fig.

91 1g). Collectively, this data indicates that NK cells are considerably affected during $S$.

92 Typhimurium infection, characterized by conversion to ILC1-like cells and organ specific

93 depletion. 
a

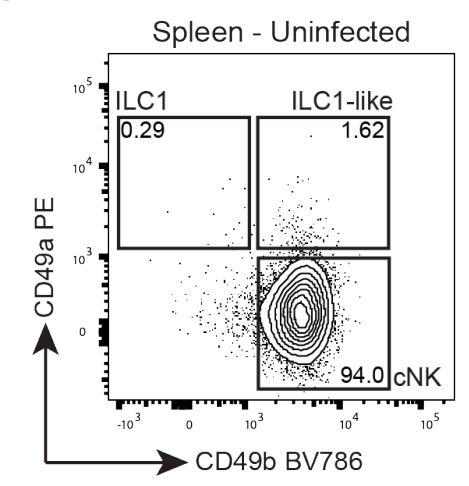

C

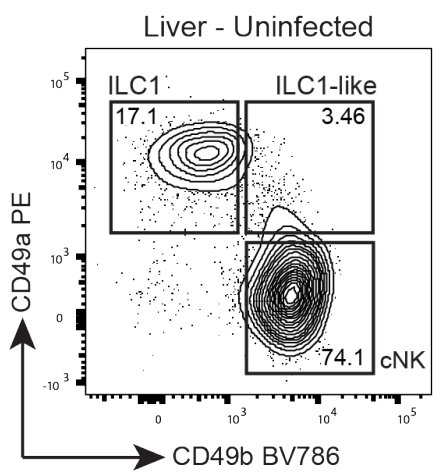

e

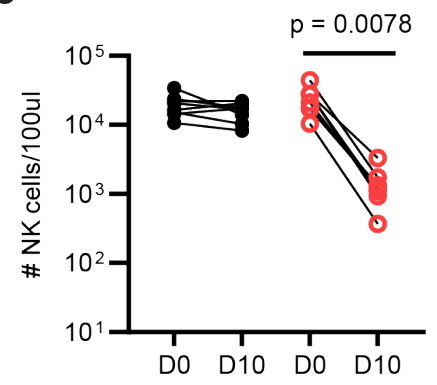

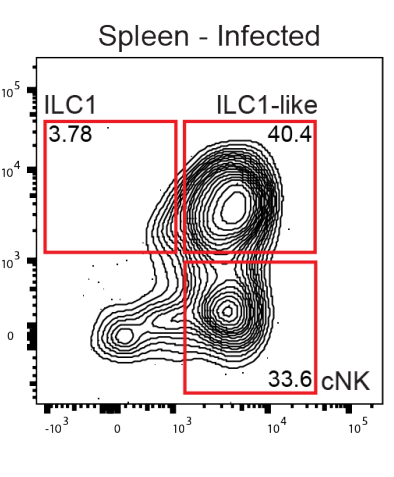

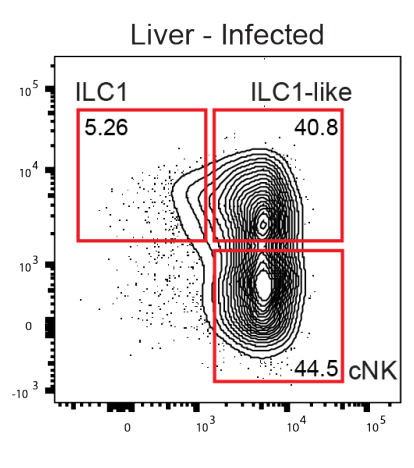

f

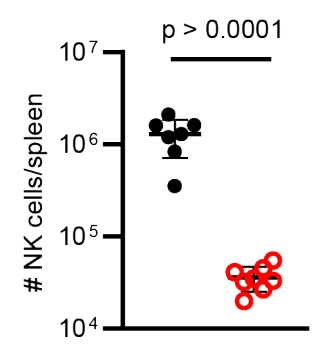

b

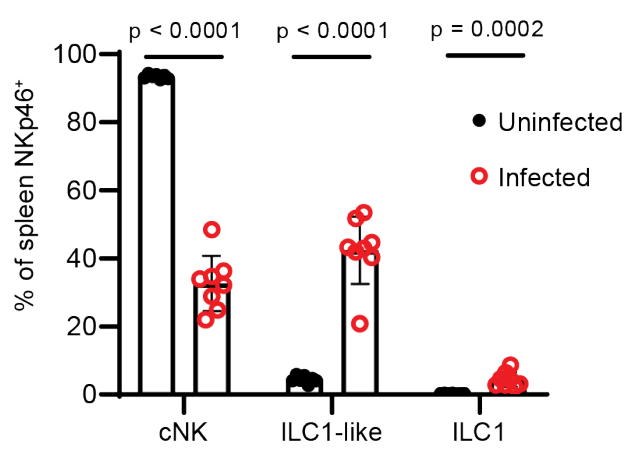

d

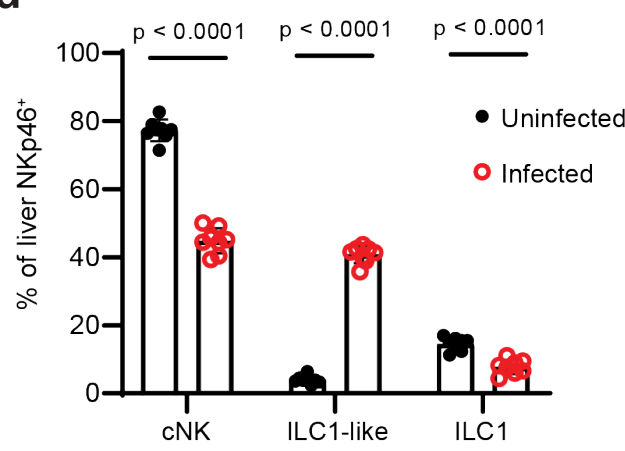

g



Figure 1: NK cells are depleted and converted to ILC1-like cells during $S$. Typhimurium infection. Group 1 ILCs were analysed by flow cytometry 10 days after infection with $S$. Typhimurium. Representative flow cytometry plots showing CD49a and CD49b expression from one uninfected and one infected mouse spleen (a) and liver (c) are shown, along with graphs displaying relative percentages of cNK, ILC1-like, and ILC1 within the spleen (b) and liver $(\mathbf{d})(\mathrm{n}=8)$. Total numbers of NK cells per $100 \mu \mathrm{l}$ of blood prior to infection and at day 10 post infection were determined by flow cytometry (e). At 10 days post infection, numbers of NK cells in the spleen (f) and liver (g) were determined by flow cytometry. Data from one experiment. Each symbol represents an individual mouse, graphs show mean value \pm SEM. Statistical $\mathrm{p}$ values determined by Mann-Whitney $t$ test, or Wilcoxon rank test for (e). No $\mathrm{p}$ value indicates no significant difference. 
Deletion of $C I S H$, but not conditional deletion of $T g f b R I I$, results in enhanced antiour infection model could be dampening the ability of NK cells to contribute to bacterial clearance. To address this, we infected mice with conditional deletion of the TGF- $\beta$ receptor II specifically within NK cells $\left(T g f b R I I^{F L}\right)$. NK cell mediated bacterial clearance could be further intracellular checkpoint molecule CIS, has been reported to enhance NK cell function and proliferation (Delconte et al., 2016, 2020). While deletion of CIS does not lead to increased NK cell accumulation in the steady state (Delconte et al., 2016), we predicted its deletion could enhance proliferation to help maintain NK cell numbers during infection. Therefore, we also infected CIS deficient mice $\left(\mathrm{Cish}^{\mathrm{KO}}\right)$. We also infected mice with CIS deficiency and conditional deletion of TGF- $\beta$ signaling within NK cells $\left(T g f b R I I^{F L} / C i s h^{K O}\right)$ to determine if these could have a synergistic effect in improving anti-bacterial immunity. Surprisingly, $T g f b R I I^{F L}$ mice did not show a reduced bacterial load in either the spleen (Fig. 2a) or the liver (Fig. 2b) compared to wild-type controls. Conversely, $C i s h^{K O}$ mice exhibited a significant reduction in bacterial burden in both organs (Fig, 2a,b). The combination of both NK cell gain of function genes did not synergize further reduce bacterial burdens (Fig, 2a,b). To investigate whether the enhanced immunity in $\operatorname{Cish}^{K O}$ mice was NK cell dependent, $C i s h^{K O}$ mice were treated with $\alpha$ NK1.1 to deplete NK cells. No differences were observed in the spleen (Figure, 2c). In the liver, while $\operatorname{Cish}^{K O}$ was able to significantly reduce bacterial burdens, $\operatorname{Cish}^{K O}$ with NK cell depletion did not significantly differ from wild-type or $C_{i s h}^{K O}$ alone (Figure, 2d).

132 Further, IL-6 was the only cytokine significantly increased in the plasma of $\mathrm{Cish}^{\mathrm{KO}}$ mice at day 
133 two compared to wild-type controls (Supplementary Figure 1), suggesting Cish deletion may

134 primarily act through myeloid cells in this case. No significant increases in cytokine levels

135 were observed at day nine post infection (Supplementary Figure 2). Thus, we found no

136 evidence that the reductions in bacterial burdens observed in the livers of $\operatorname{Cish}^{K O}$ mice was due

137 to enhancement of NK cell function. 
a

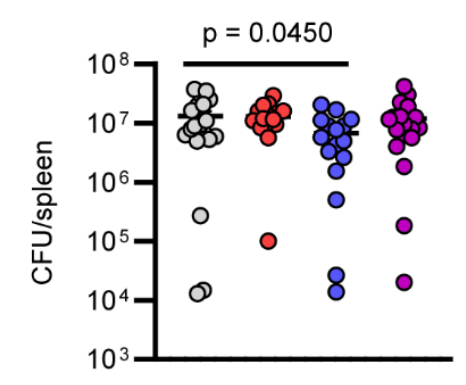

C

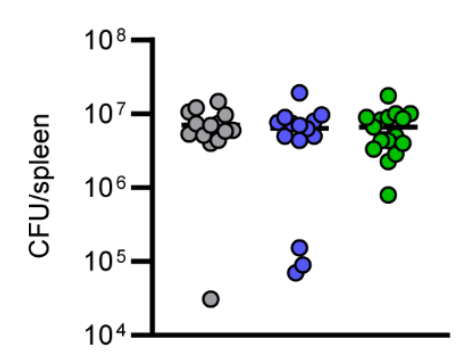

b

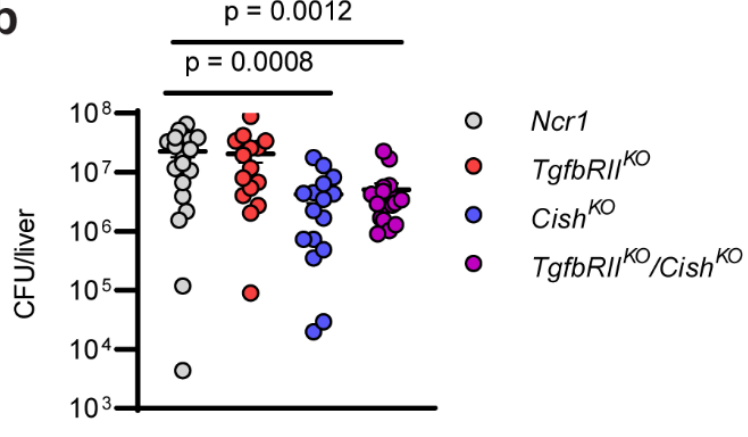

d

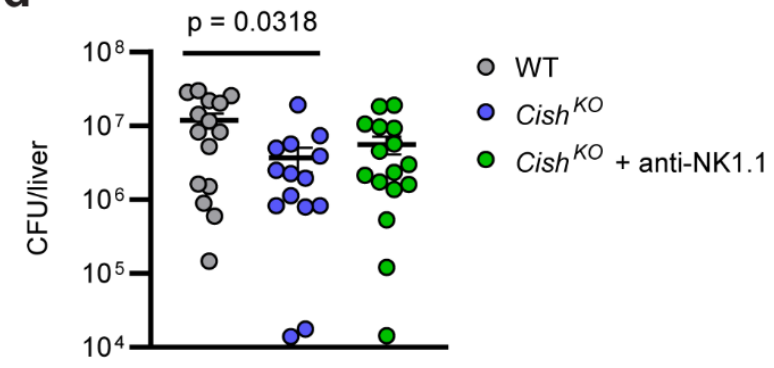

140 Figure 2: Deletion of $\boldsymbol{C I S H}$, but not conditional deletion of TGF- $\beta$ signalling, results in

141 reduced bacterial burdens. $N c r l C r e, T g f b R I I^{F L}, C i s h^{K O}$, and $T g f b R I I^{F L} / C i s h^{K O}$ mice were infected with $S$. Typhimurium. At day 10 post infection, spleens and livers were collected to quantify bacterial burden. Colony forming units (CFU) per spleen (a) and liver (b) are shown (n=15-19). Data from two independent experiments. C57BL/6 and Cish ${ }^{K O}$ were infected while simultaneously treated with either anti-NK1.1 depletion antibody, or isotype control. Colony forming units per spleen $(\mathbf{c})$ and liver $(\mathbf{d})$ are shown $(\mathrm{n}=14-16)$. Data from two independent experiments. Each symbol represents an individual mouse, graphs show mean value \pm SEM. Statistical $\mathrm{p}$ values determined by Mann-Whitney $t$ test, where no $\mathrm{p}$ value indicates no significant difference. 

deletion of TGF- $\beta$ signaling within NK cells did not prevent conversion to ILC1-like cells in either the spleen (Fig. 3a,b) or liver (Fig. 3c,d) of infected mice. We also targeted TGF- $\beta$ signaling therapeutically using the TGF- $\beta$ receptor 1 kinase inhibitor galunisertib in infected change (Supplemental Fig. 3c), or NK cell to ILC1-like conversion (Supplemental Fig. 3d,e) in galunisertib treated mice compared to untreated controls. Further, neither conditional deletion of $T g f b r I I$, deletion of Cish, or a combination were able to prevent NK cell lymphopenia observed in the blood and spleen of infected mice (data not shown). Taken together, this data suggests that canonical TGF- $\beta$ / TGF-bRII signaling is redundant in driving NK cell to ILC1-like conversion during $S$. Typhimurium infection. This may explain why no 172 reduction in bacterial burdens were observed in $T g f b R I I^{F L}$ mice or mice treated with the TGF-

173 bRI inhibitor galunisertib, although whether the conversion limits NK-mediated immunity during bacterial infection remains unclear. 
a

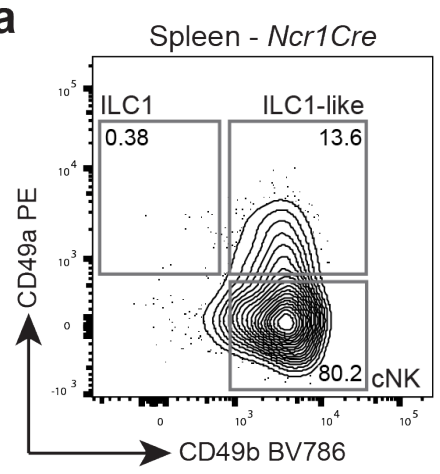

C

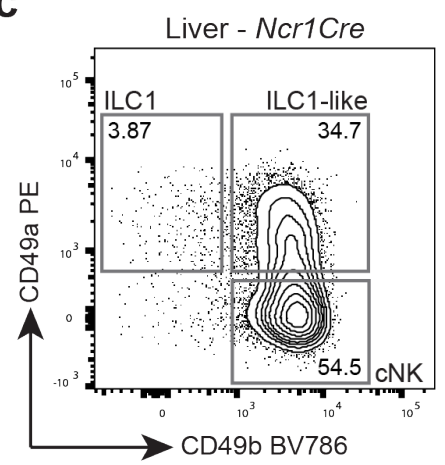

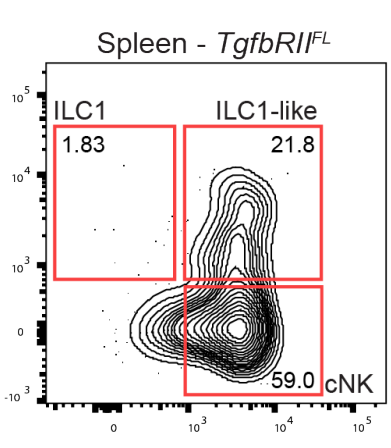

b
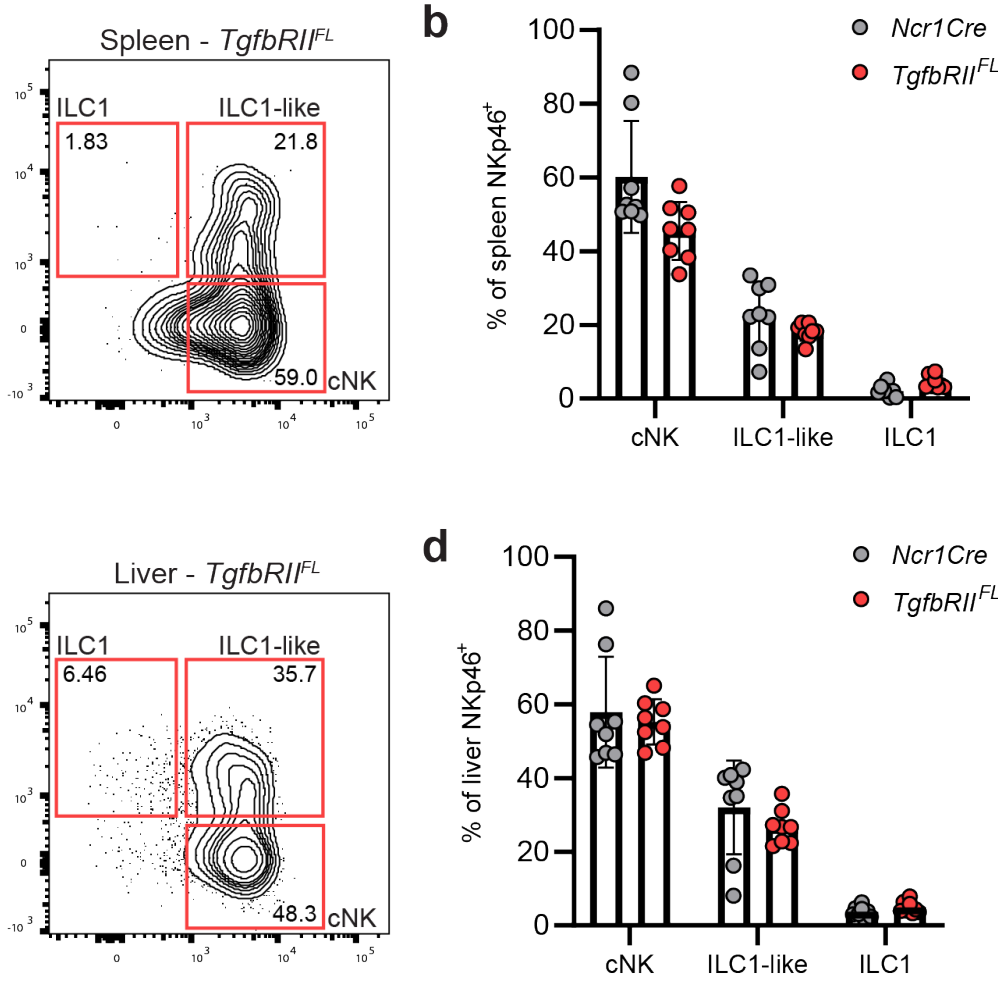

d

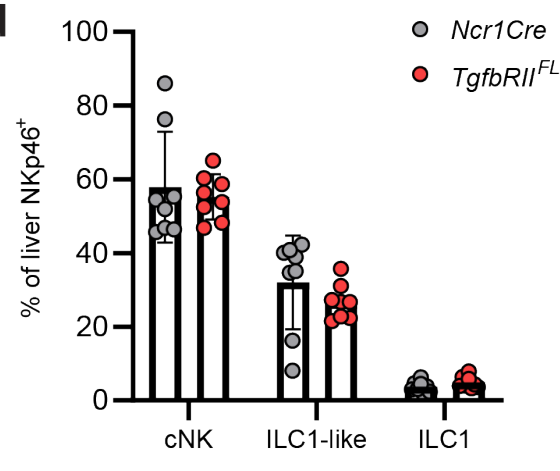

Figure 3: TGF- $\beta$ signalling is redundant in NK to ILC1-like plasticity in $S$. Tyhpimurium infection. $T g f b R I I^{F L}$ mice, which lack the TGF- $\beta$ receptor specifically within NK cells, as well as $N$ cr 1 Cre controls, were infected with $S$. Typhimurium. At day 10 post infection, Group 1 ILCs were analysed by flow cytometry. Representative flow cytometry plots showing CD49a and CD49b expression from one NcrlCre and one $T g f b R I I^{F L}$ mouse spleen (a) and liver (c) are shown, along with graphs displaying relative percentages of cNK, ILC1-like, and ILC1 within the spleen (b) and liver (d) $(n=8)$. Data from one experiment. Each symbol represents an individual mouse, graphs show mean value \pm SEM. Statistical $p$ values determined by MannWhitney $t$ test, where no $\mathrm{p}$ value indicates no significant difference. 


\section{Discussion}

The purpose of this study was to gain a better understanding of how NK cells are their efficacy as a target during acute or chronic bacterial infection is unknown. Immunotherapy for treating bacterial infections, particularly in the case of antibiotic resistance, is an emerging field (McCulloch et al., 2021), for which NK-mediated immunotherapy may also show potential. Identification of specific regulatory molecules and checkpoints acting on NK cells during bacterial infection could uncover novel immunotherapy targets to enhance NK-mediated bacterial immunity. was not unexpected. This conversion has previously been observed in other diseases including cancer (Gao et al., 2017) and parasitic infection (Park et al., 2019). Further, Salmonella (or M2-like) phenotype (Stapels et al., 2018), promoting the production of regulatory cytokines such as IL-10 and TGF- $\beta$ (Jaslow et al., 2018). However, by using transgenic mice and therapeutic inhibition, we found that conversion to ILC1-like cells was likely not TGF- $\beta$ dependent in our model. The TGF- $\beta$ superfamily is a large group of over 33 regulatory proteins which have both distinct and overlapping functions (Morikawa et al., 2016). Therefore it is reasonable to assume that other members of the TGF- $\beta$ superfamily could also drive this conversion, which has already been observed in the case of Activin A (Rautela et al., 2019).

209 Our findings using the therapeutic inhibitor galunisertib, which targets the TGF- $\beta$ receptor 1

210 kinase, may also be hindered by inconsistencies in the literature on the appropriate dosing of 
every second day by i.p. injection (Rautela et al., 2019) to $150 \mathrm{mg} / \mathrm{kg}$ twice daily by oral gavage

213 (Gunderson et al., 2020). Whether underdosing was a defining factor in our results is unknown.

214 The driving factor, or factors, behind NK to ILC1-like conversion would need to be identified before definitively determining whether this conversion limits anti-bacterial immunity. infection. CIS is a suppressor of cytokine signaling (SOCS) protein which functions as a negative regulator of cytokine signaling. In the case of NK cells, IL-15 signaling leads to phosphorylation of Janus kinase (JAK)1 and 3, and subsequent recruitment and phosphorylation of STAT5. This allows STAT5 to then translocate to the nucleus to transcribe variety of genes associated with NK cell function and survival (Ma et al., 2006). Also transcribed is Cish, encoding CIS, which binds to JAK1 and JAK3, targeting them for proteasomal degradation. In this way, CIS acts as a negative feedback loop to limit IL-15 signaling and NK cell activation (Delconte et al., 2016). However, STAT5 signaling is also active in other cell types in response to signaling by cytokines other than IL-15, including IL2 and GM-CSF. CIS also inhibits T cell receptor signaling (Palmer et al., 2015). Thus, CIS has also been shown to regulate additional immune cells including ILC2s (Kotas et al., 2021), $\mathrm{CD}^{+}$and CD8 ${ }^{+}$T cells (Palmer et al., 2015; Yang et al., 2013), neutrophils (Louis et al., 2020), and macrophages (Carow et al., 2017; E. et al., 2021). Considering this was a whole mouse knockout of CIS, we cannot be sure of which cell type, or combinations of cell types, benefited from CIS deletion to promote anti-bacterial immunity. We found no evidence to confirm our initial hypothesis that CIS deletion acted through NK cell enhancement. Curiously, our results may be somewhat conflicting with other studies. Despite being a negative regulator of immune function, CIS has previously been shown to mediate early control of tuberculosis infection in a mouse model (Carow et al., 2017). In humans, single nucleotide 
polymorphisms (SNPs) in Cish have been associated with increased susceptibility to

237 tuberculosis, malaria, and bacteremia (Khor et al., 2010). However, the functional implications of these SNPs on immune response were not established in the study. These previous results may be explained by multiple observations in which excessive inflammation worsens

240 infectious disease. This can be epitomized by the curious case of anti-PD1 therapy in tuberculosis, which exacerbates disease severity and reactivates latent infection (Barber et al., 2019; Kauffman et al., 2021). Thus, in the case of tuberculosis, immune enhancement does not always lead to greater immunity. That we have found CIS deletion enhances anti-Salmonella

244 immunity, where others have found it impedes anti-tuberculosis immunity, may be explained by underlying differences in the physiology and pathology of these two pathogens. These differences must be rigorously examined if any pharmacological targeting of CIS signaling is to be trialed to treat bacterial infections. infection. However, the precise driver of conversion as well as the functional relevance this plasticity has on infection outcome remains elusive. Here, we have also shown that CIS is a potent immune checkpoint in anti-Salmonella immunity. However, expression of CIS is also conserved across other immune cell types such as myeloid cells, and thus the exact cell type or mechanism where CIS acts on to restrict bacterial clearance could not be addressed in this current study. Future work is warranted to elucidate how CIS inhibition enacts its effects, and whether pharmacological inhibition of this molecule could enhance anti-bacterial immunity. 


\section{Methods}

259

260

261

262

263

264

265

266

267

268

269

270

271

272

273

\section{Mouse models}

$N c r l^{c r e / w t} T g f b R I I^{f l f l}$ mice were used as conditional TgfbRII-deficiency specific to NK cells, obtained by crossing as previous described by our group (Gao et al., 2017). Cish ${ }^{-/}$were maintained on a C57BL/6 background (Delconte et al., 2016). To obtain a double deficient mouse model, $N c r 1^{c r e / w t} T g f b R I f^{f l f l}$ were back crossed with $\mathrm{Cish}^{-/-}$to obtain a $\mathrm{Cish}^{-/-}$ $N c r 1^{c r e / w t} T g f b R I I^{f l f l}$ mice strain. $N c r 1^{c r e / w t}$ mice were considered wild-type controls for some experiments. $\mathrm{Ncr} \mathrm{I}^{\mathrm{cre} / \mathrm{cre}} \mathrm{Mcl} \mathrm{I}^{\mathrm{fl} f \mathrm{fl}}$ mice were used as NK cell deficient controls (Sathe et al., 2014). All experiments were performed using cells from age/sex matched cohort of mice (age range 8-12 weeks). Cohort sizes are described in each figure legends, where power calculations were used to estimated sample size needed to achieve statistical significance at a $50 \%$ change in bacterial burden or immune parameters with a power of 0.80 and Type I error (alpha) of 0.05. For infection studies, mice with no detectable bacterial load at the end of the experiment were considered to have not taken up infection and were excluded from further analysis. All experiments were approved by the University of Queensland's Animal Ethics Committees.

\section{Bacterial strains and in vivo infections}

Mice were infected with an attenuated aroA mutant strain of Salmonella enterica serovar Typhimurium, SL3261 (Hoiseth \& Stocker, 1981). For in vivo infection, bacteria were grown at $37^{\circ} \mathrm{C}$ with shaking in Lysogeny broth (LB) for 16 to 18 hours. $\mathrm{OD}_{600}$ was used to enumerate bacteria, before being diluted to the appropriate concentration in PBS. Mice were infected by intraperitoneal (i.p) injection with $5 \times 10^{6}$ colony forming units (CFU) of SL3261 in $200 \mu 1$ and sacrificed at the described times post-infection. The TGF- $\beta$ receptor I inhibitor galunisertib (LY2157299, SelleckChem, Houston TX) was given at a dose of $10 \mathrm{mg} / \mathrm{kg}$ by i.p infection, as described previously (Rautela et al., 2019). To deplete NK cells, appropriate mice 
bioRxiv preprint doi: https://doi.org/10.1101/2021.11.29.470332; this version posted November 29,2021 . The copyright holder for this preprint (which was not certified by peer review) is the author/funder, who has granted bioRxiv a license to display the preprint in perpetuity. It is made available under aCC-BY 4.0 International license.

were treated with $100 \mu \mathrm{g}$ of anti-NK1.1 antibody (PK136, BioXCell, Lebanon NH) or isotype control (2AE, BioXCell) on days $-3,0,3$, and 8 relative to infection.

\section{Murine tissue collection}

Blood samples were taken from mice by retro-orbital bleeds into EDTA-coated tubes. Tubes were centrifuged at 1,500 $\mathrm{g}$ for 15 minutes, and plasma removed from cell pellet. Plasma samples were stored at $-20^{\circ} \mathrm{C}$ until analysis. At the experimental endpoint, mice were euthanized by $\mathrm{CO}_{2}$ asphyxiation. Spleens and livers were dissected and held in PBS until processing. Bacterial counts were enumerated from organs by homogenizing samples in $0.1 \%$ Triton-X (Sigma-Aldrich, Burlington MA) in PBS before serially diluting in PBS and plating on LB agar plates.

\section{Flow cytometry}

Spleens and livers were passed through a $70 \mu \mathrm{m}$ or $100 \mu \mathrm{m}$ cell strainer, respectively, in cold fluorescence-activated cell sorting (FACS) buffer (PBS containing $2 \%$ fetal bovine serum and 2mM EDTA). Leukocytes were enriched using 37.5\% Percoll solution (GE Healthcare, Uppsala, Sweden)) and red blood cells lysed with Ammonium-Chloride-Potassium (ACK) lysis buffer (Biolgend, San Diego CA). Dead cells were stained with Fixable Viability Stain 440UV (1:1000 in PBS, Becton Dickinson, Franklin Lakes NJ) for 15 minutes at room temperature. Fc receptors were blocked by incubation for 15 minutes in Fc Blocking Reagent (1:100 in FACS buffer, Miltenyi Biotec, Bergisch Gladbach, Germany). Single-cell suspensions were stained with the indicated fluorescent antibodies on ice for 45 minutes. For intracellular cytokine staining, cells were fixed and permeabilized using the FoxP3/Transcription Factor Staining Buffer Set (eBioscience, San Diego CA), then stained for 
309 (HA31/8) were purchased from Becton Dickinson (Franklin Lakes NK). Antibodies targeting CD226 (TX42.1), CD335 (29A1.4), KLRG1 (2F1/KLRG1), CD19 (6D5), Ly6G (1A8), F4/80 (BM8), and CD62L (MEL-14) were purchased from Biolegend. Antibodies targeting Tim-3 (RMT3-23), Eomes (Dan11mag), and FoxP3 (FJK-16S) were purchased from eBioscience (San Diego CA).

\section{Measurement of cytokines}

IFN- $\gamma$ titers were determined from murine serum samples using a Mouse IFN- $\gamma$ ELISA set (Becton Dickinson) as per the manufacturer's instructions. Other cytokines were determined using Cytometric Bead Array (Becton Dickinson) as per the manufacturer's instructions.

\section{Statistics}

324 figure legends, and error bars represent SEM. Levels of statistical significance are expressed as $p$ values. 


\section{Conflict of Interest}

329 The authors declare that the research was conducted in the absence of any commercial or

330 financial relationships that could be construed as a potential conflict of interest.

\section{Author Contributions}

332 T.R.M. and F.S.F.G. designed research and wrote the paper. T.R.M., and G.R.R., performed research and analysed data. T.J.W and F.S.F.G. supervised work.

\section{Funding}

335 This work is supported by project grants from the National Health and Medical Research 336 Council (NHMRC) of Australia (\#1140406 to F.S.F.G). F.S.F.G is funded by a UQ Diamantina 337 Institute Laboratory Start-Up Package, a US Department of Defense - Breast Cancer Research 338 Program - Breakthrough Award Level 1 (\#BC200025), a ANZSA SRG Grant, and was 339 supported by a grant (1158085) awarded through the Priority-driven Collaborative Cancer

340 Research Scheme and co-funded by Cancer Australia and Cure Cancer.

\section{Acknowledgments}

342 We thank all the members of the Guimaraes and Wells laboratories; Prof. N. D. Huntington, 343 Prof. G. T. Belz, Prof. S. Bell, Prof. M. Sweet, and Prof. A. Yoshimura for discussion, 344 comments, and advice in this project; Prof. E. Vivier for providing the NKp46cre mice; and 345 Profs. J. Ihle and E. Parganas for providing the CIS knockout mice. This research was carried 346 out at the Translational Research Institute, Woolloongabba, QLD 4102, Australia. The 347 Translational Research Institute is supported by grants from the Australian and Queensland 348 Governments. 
351

352

353

354

355

356

357

358

359

360

361

362

363

364

365

366

367

368

369

370

371

372

373

374

375

376

377

378

379

380

381

\section{References}

Barber, D. L., Sakai, S., Kudchadkar, R. R., Fling, S. P., Day, T. A., Vergara, J. A., Ashkin, D., Cheng, J. H., Lundgren, L. M., Raabe, V. N., Kraft, C. S., Nieva, J. J., Cheever, M. A., Nghiem, P. T., \& Sharon, E. (2019). Tuberculosis following PD-1 blockade for cancer immunotherapy. Science Translational Medicine, 11(475), 1-8. https://doi.org/10.1126/scitranslmed.aat2702

Carow, B., Gao, Y., Terán, G., Yang, X. O., Dong, C., Yoshimura, A., \& Rottenberg, M. E. (2017). CISH controls bacterial burden early after infection with Mycobacterium tuberculosis in mice. Tuberculosis. https://doi.org/10.1016/j.tube.2017.09.007

Delconte, R. B., Guittard, G., Goh, W., Hediyeh-Zadeh, S., Hennessy, R. J., Rautela, J., Davis, M. J., Souza-Fonseca-Guimaraes, F., Nunès, J. A., \& Huntington, N. D. (2020). NK Cell Priming From Endogenous Homeostatic Signals Is Modulated by CIS. Frontiers in Immunology, 11. https://doi.org/10.3389/fimmu.2020.00075

Delconte, R. B., Kolesnik, T. B., Dagley, L. F., Rautela, J., Shi, W., Putz, E. M., Stannard, K., Zhang, J. G., Teh, C., Firth, M., Ushiki, T., Andoniou, C. E., Degli-Esposti, M. A., Sharp, P. P., Sanvitale, C. E., Infusini, G., Liau, N. P. D., Linossi, E. M., Burns, C. J., ... Huntington, N. D. (2016). CIS is a potent checkpoint in NK cell-mediated tumor immunity. Nature Immunology. https://doi.org/10.1038/ni.3470

E., S. K., Neha, C., M., C. Y., A., M. B., K., C. V., A., C. J., Harinder, S., \& A., G. R. (2021). $\mathrm{CISH}$ attenuates homeostatic cytokine signaling to promote lung-specific macrophage programming and function. Science Signaling, 14(698), eabe5137. https://doi.org/10.1126/scisignal.abe5137

Gao, Y., Souza-Fonseca-Guimaraes, F., Bald, T., Ng, S. S., Young, A., Ngiow, S. F., Rautela, J., Straube, J., Waddell, N., Blake, S. J., Yan, J., Bartholin, L., Lee, J. S., Vivier, E., Takeda, K., Messaoudene, M., Zitvogel, L., Teng, M. W. L., Belz, G. T., ... Smyth, M. J. (2017). Tumor immunoevasion by the conversion of effector NK cells into type 1 innate lymphoid cells. Nature Immunology, 18(9), 1004-1015. https://doi.org/10.1038/ni.3800

Gunderson, A. J., Yamazaki, T., McCarty, K., Fox, N., Phillips, M., Alice, A., Blair, T., Whiteford, M., O’Brien, D., Ahmad, R., Kiely, M. X., Hayman, A., Crocenzi, T., Gough, M. J., Crittenden, M. R., \& Young, K. H. (2020). TGF $\beta$ suppresses CD8+ T cell expression of CXCR3 and tumor trafficking. Nature Communications. 
383

Hawke, L. G., Mitchell, B. Z., \& Ormiston, M. L. (2020). TGF- $\beta$ and IL-15 Synergize through MAPK Pathways to Drive the Conversion of Human NK Cells to an Innate Lymphoid Cell 1-like Phenotype. The Journal of Immunology, 204(12), 3171-3181. https://doi.org/10.4049/jimmunol.1900866

Hoiseth, S. K., \& Stocker, B. A. D. (1981). Aromatic-dependent Salmonella typhimurium are non-virulent and effective as live vaccines. Nature. https://doi.org/10.1038/291238a0

Jaslow, S. L., Gibbs, K. D., Fricke, W. F., Wang, L., Pittman, K. J., Mammel, M. K., Thaden, J. T., Fowler, V. G., Hammer, G. E., Elfenbein, J. R., \& Ko, D. C. (2018). Salmonella Activation of STAT3 Signaling by SarA Effector Promotes Intracellular Replication and Production of IL-10. Cell Reports, 23(12), 3525-3536. https://doi.org/10.1016/j.celrep.2018.05.072

Kauffman, K. D., Sakai, S., Lora, N. E., Namasivayam, S., Baker, P. J., Kamenyeva, O., Foreman, T. W., Nelson, C. E., Oliveira-de-Souza, D., Vinhaes, C. L., Yaniv, Z., Lindestam Arleham, C. S., Sette, A., Freeman, G. J., Moore, R., Sher, A., Mayer-Barber, K. D., Andrade, B. B., Kabat, J., ... Barber, D. L. (2021). PD-1 blockade exacerbates Mycobacterium tuberculosis infection in rhesus macaques. Science Immunology, 6(55), eabf3861. https://doi.org/10.1126/sciimmunol.abf3861

Khor, C. C., Vannberg, F. O., Chapman, S. J., Guo, H., Wong, S. H., Walley, A. J., Vukcevic, D., Rautanen, A., Mills, T. C., Chang, K.-C., Kam, K.-M., Crampin, A. C., Ngwira, B., Leung, C.-C., Tam, C.-M., Chan, C.-Y., Sung, J. J. Y., Yew, W.-W., Toh, K.-Y., ... Hill, A. V. S. (2010). CISH and Susceptibility to Infectious Diseases . New England Journal of Medicine. https://doi.org/10.1056/nejmoa0905606

Kotas, M. E., Mroz, N. M., Koga, S., Liang, H. E., Schroeder, A. W., Ricardo-Gonzalez, R. R., Schneider, C., \& Locksley, R. M. (2021). CISH constrains the tuft-ILC2 circuit to set epithelial and immune tone. Mucosal Immunology. https://doi.org/10.1038/s41385-02100430-6

Kupz, A., Scott, T. A., Belz, G. T., Andrews, D. M., Greyer, M., Lew, A. M., Brooks, A. G., Smyth, M. J., Curtiss, R., Bedoui, S., \& Strugnell, R. A. (2013). Contribution of Thy1+ NK cells to protective IFN- $\gamma$ production during Salmonella Typhimurium infections. Proceedings of the National Academy of Sciences of the United States of America, 110(6), 
414

415

416

417

418

419

420

421

422

423

424

425

426

427

428

429

430

431

432

433

434

435

436

437

438

439

440

441

442

Louis, C., Souza-Fonseca-Guimaraes, F., Yang, Y., D’Silva, D., Kratina, T., Dagley, L., Hediyeh-Zadeh, S., Rautela, J., Masters, S. L., Davis, M. J., Babon, J. J., Ciric, B., Vivier, E., Alexander, W. S., Huntington, N. D., \& Wicks, I. P. (2020). NK cell-derived GMCSF potentiates inflammatory arthritis and is negatively regulated by CIS. Journal of Experimental Medicine, 217(5). https://doi.org/10.1084/jem.20191421

Ma, A., Koka, R., \& Burkett, P. (2006). Diverse functions of IL-2, IL-15, and IL-7 in lymphoid homeostasis. Annual Review of Immunology, 24, 657-679. https://doi.org/10.1146/annurev.immunol.24.021605.090727

McCulloch, T. R., Wells, T. J., \& Souza-Fonseca-Guimaraes, F. (2021). Towards efficient immunotherapy for bacterial infection. In Trends in Microbiology (pp. 1-12). https://doi.org/10.1016/j.tim.2021.05.005

Morikawa, M., Derynck, R., \& Miyazono, K. (2016). TGF- $\beta$ and the TGF- $\beta$ family: Contextdependent roles in cell and tissue physiology. In Cold Spring Harbor Perspectives in Biology. https://doi.org/10.1101/cshperspect.a021873

Palmer, D. C., Guittard, G. C., Franco, Z., Crompton, J. G., Eil, R. L., Patel, S. J., Ji, Y., Van Panhuys, N., Klebanoff, C. A., Sukumar, M., Clever, D., Chichura, A., Roychoudhuri, R., Varma, R., Wang, E., Gattinoni, L., Marincola, F. M., Balagopalan, L., Samelson, L. E., \& Restifo, N. P. (2015). Cish actively silences TCR signaling in CD8+ T cells to maintain tumor tolerance. Journal of Experimental Medicine. https://doi.org/10.1084/jem.20150304

Park, E., Patel, S., Wang, Q., Andhey, P., Zaitsev, K., Porter, S., Hershey, M., Bern, M., Plougastel-Douglas, B., Collins, P., Colonna, M., Murphy, K. M., Oltz, E., Artyomov, M., Sibley, L. D., \& Yokoyama, W. M. (2019). Toxoplasma gondii infection drives conversion of NK cells into ILC1-like cells. ELife. https://doi.org/10.7554/eLife.47605

Rautela, J., Dagley, L. F., De Oliveira, C. C., Schuster, I. S., Hediyeh-Zadeh, S., Delconte, R. B., Cursons, J., Hennessy, R., Hutchinson, D. S., Harrison, C., Kita, B., Vivier, E., Webb, A. I., Degli-Esposti, M. A., Davis, M. J., Huntington, N. D., \& Souza-Fonseca-Guimaraes, F. (2019). Therapeutic blockade of activin-A improves NK cell function and antitumor immunity. Science Signaling, 12(596). https://doi.org/10.1126/scisignal.aat7527 
443

444

445

446

447

448

449

450

451

452

453

454

455

456

457

458

459

460

461

462

463

464

465

466

467

468

469

470

471

472

Robert, C. (2020). A decade of immune-checkpoint inhibitors in cancer therapy. Nature Communications, 11(1), 3801. https://doi.org/10.1038/s41467-020-17670-y

Sathe, P., Delconte, R. B., Souza-Fonseca-Guimaraes, F., Seillet, C., Chopin, M., Vandenberg, C. J., Rankin, L. C., Mielke, L. A., Vikstrom, I., Kolesnik, T. B., Nicholson, S. E., Vivier, E., Smyth, M. J., Nutt, S. L., Glaser, S. P., Strasser, A., Belz, G. T., Carotta, S., \& Huntington, N. D. (2014). Innate immunodeficiency following genetic ablation of Mcl1 in natural killer cells. Nature Communications, 5, 4539. https://doi.org/10.1038/ncomms5539

Souza-Fonseca-Guimaraes, F., Cursons, J., \& Huntington, N. D. (2019). The Emergence of Natural Killer Cells as a Major Target in Cancer Immunotherapy. In Trends in Immunology. https://doi.org/10.1016/j.it.2018.12.003

Stapels, D. A. C., Hill, P. W. S., Westermann, A. J., Fisher, R. A., Thurston, T. L., Saliba, A. E., Blommestein, I., Vogel, J., \& Helaine, S. (2018). Salmonella persisters undermine host immune defenses during antibiotic treatment. Science, 362(6419), 1156-1160. https://doi.org/10.1126/science.aat7148

Vély, F., Barlogis, V., Vallentin, B., Neven, B., Piperoglou, C., Ebbo, M., Perchet, T., Petit, M., Yessaad, N., Touzot, F., Bruneau, J., Mahlaoui, N., Zucchini, N., Farnarier, C., Michel, G., Moshous, D., Blanche, S., Dujardin, A., Spits, H., ... Vivier, E. (2016). Evidence of innate lymphoid cell redundancy in humans. Nature Immunology, 17(11), 1291-1299. https://doi.org/10.1038/ni.3553

Viel, S., Marcais, A., Guimaraes, F. S.-F., Loftus, R., Rabilloud, J., Grau, M., Degouve, S., Djebali, S., Sanlaville, A., Charrier, E., Bienvenu, J., Marie, J. C., Caux, C., Marvel, J., Town, L., Huntington, N. D., Bartholin, L., Finlay, D., Smyth, M. J., \& Walzer, T. (2016). TGF-beta inhibits the activation and functions of NK cells by repressing the mTOR pathway. Science Signaling, 9(415), ra19. https://doi.org/10.1126/scisignal.aad1884

Yang, X. O., Zhang, H., Kim, B. S., Niu, X., Peng, J., Chen, Y., Kerketta, R., Lee, Y. H., Chang, S. H., Corry, D. B., Wang, D., Watowich, S. S., \& Dong, C. (2013). The signaling suppressor CIS controls proallergic T cell development and allergic airway inflammation. Nature Immunology. https://doi.org/10.1038/ni.2633 
a

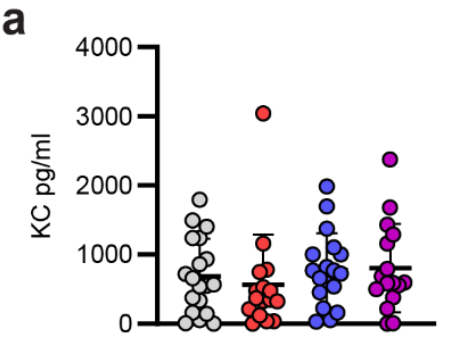

d

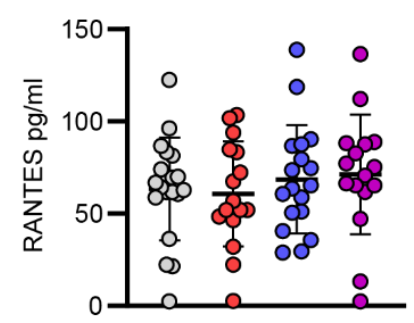

g

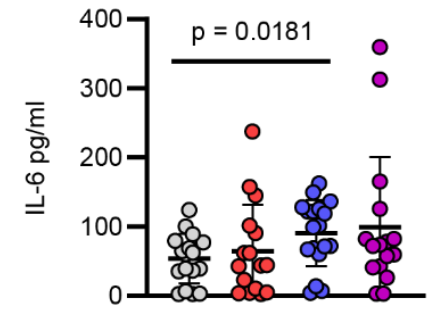

b

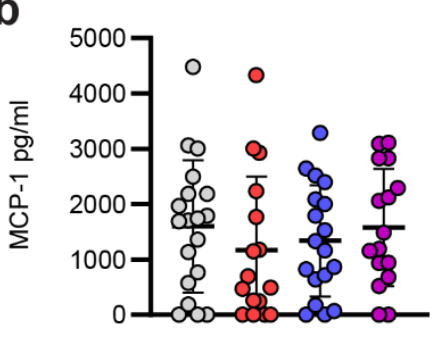

e

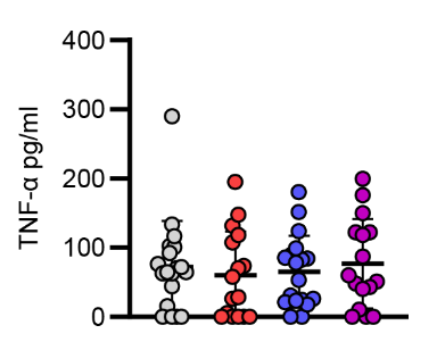

C

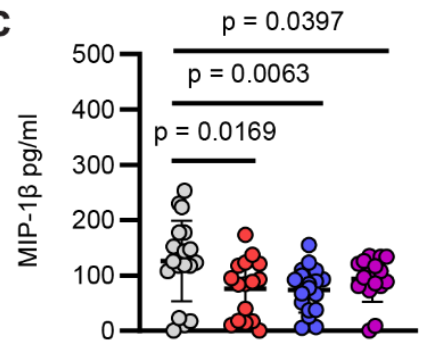

h

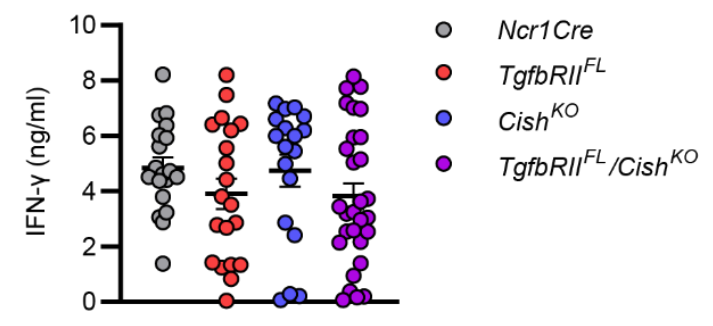

Figure 2 - figure supplement 1: Plasma cytokine levels in transgenic mice at day 2 post infection. NcrlCre, TgfbRII ${ }^{F L}, C i s h^{K O}$, and $T g f b R I I^{F L} / C i s h^{K O}$ mice were infected with $S$. Typhimurium. At day 2, plasma was taken to measure cytokine levels by CBA (a-g) or ELISA (h). Levels of KC (a), MCP-1 (b), MIP-1 $\beta$ (c), RANTES (d), TNF- $\alpha$ (e), G-CSF (f), IL-6 (g), and IFN- $\gamma(\mathbf{h})$ are shown. Data from two individual experiments. Each symbol represents an individual mouse, graphs show mean value \pm SEM. Statistical $p$ values determined by MannWhitney $t$ test, where no $\mathrm{p}$ value indicates no significant difference. 
a

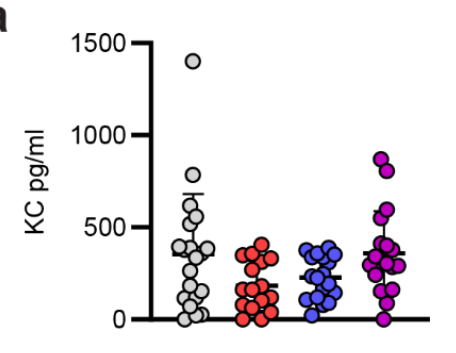

d

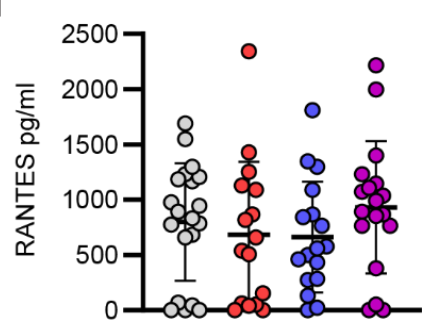

g

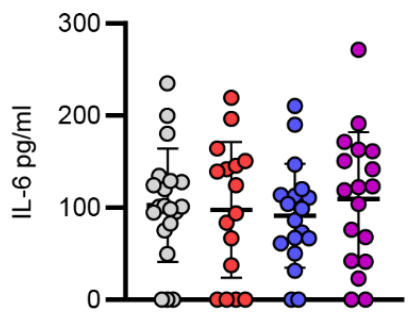

b

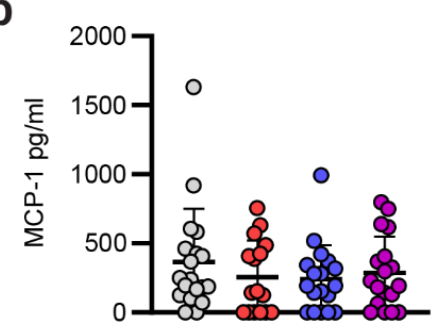

e

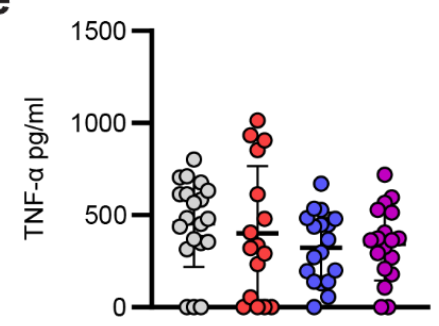

C

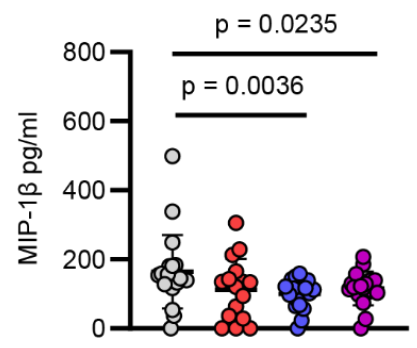

f

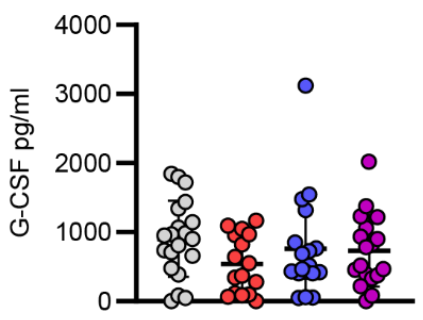

483

h



Figure 2 - figure supplement 2: Plasma cytokine levels in transgenic mice at day 9 post infection. NcrlCre, TgfbRII ${ }^{F L}, C i s h^{K O}$, and $T g f b R I I^{F L} / C i s h^{K O}$ mice were infected with $S$. Typhimurium. At day 9, plasma was taken to measure cytokine levels by CBA (a-g) or ELISA (h). Levels of KC (a), MCP-1 (b), MIP-1 $\beta$ (c), RANTES (d), TNF- $\alpha$ (e), G-CSF (f), IL-6 (g), and IFN- $\gamma(\mathbf{h})$ are shown. Data from two individual experiments. Each symbol represents an individual mouse, graphs show mean value \pm SEM. Statistical $p$ values determined by MannWhitney $t$ test, where no $\mathrm{p}$ value indicates no significant difference. 
b

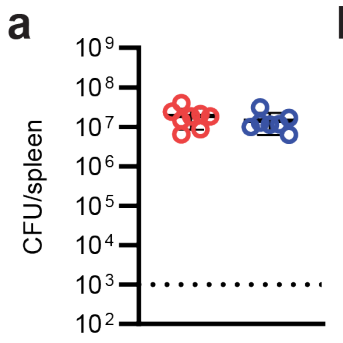

b

d

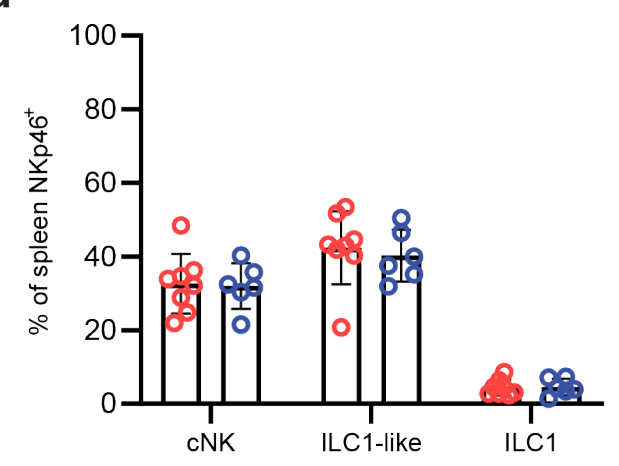

- Untreated - Galunisertib

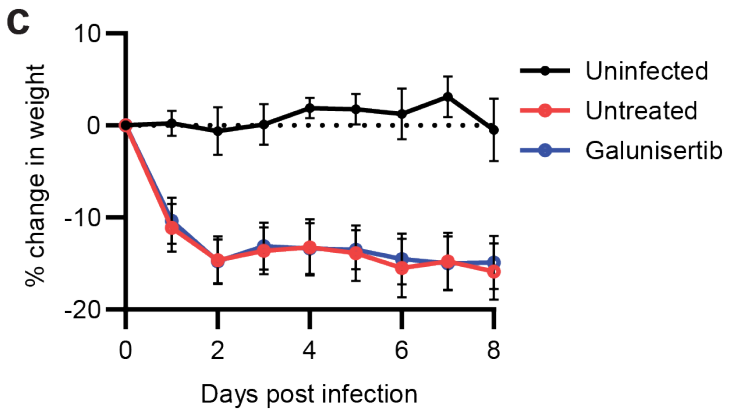

e

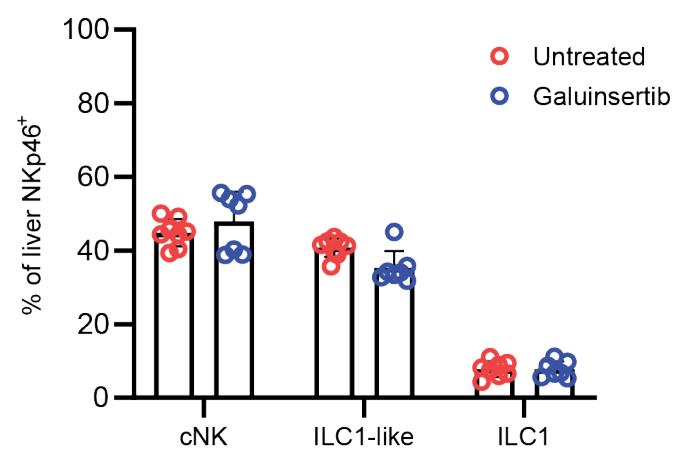

Figure 3 - figure supplement 1: Galunisertib has no effect on $\boldsymbol{S}$. Typhimurium infection. C57BL/6 mice were infected with $S$. Typhimurium and treated every second day with the TGF$\beta$ antagonist, galunisertib. At day 10 , spleens and livers were removed to quantify bacterial burdens and immune parameters. CFU per spleen (a) and liver (b) are shown, along with \% change in weight over the course of infection (c). Flow cytometry was performed to determine relative percentages of $\mathrm{cNK}$, ILC1-like, or ILC1 within $\mathrm{NKp} 46^{+}$cells in the spleen (d) and liver $(\mathbf{e})(\mathrm{n}=7-8)$. Data from one experiment. Each symbol represents an individual mouse, graphs show mean value \pm SEM. Statistical $p$ values determined by Mann-Whitney $t$ test, where no $p$ value indicates no significant difference. 